\title{
Consumo foliar da traça-das-crucíferas em couve e brócolis tratados com sinigrina
}

\section{Leaf area consumed by diamondback moth feeding on kale and brocolis treated with sinigrin}

\section{Sergio Antonio De Bortoli ${ }^{1 *}$, Jackeline da Silva Carvalho', Alessandra Marieli Vacari ${ }^{1}$, Roberto Marchi Goulart ${ }^{1}$}

RESUMO: A estimativa da área foliar, em função da sua redução causada pelo consumo por insetos filófagos, é uma metodologia básica em várias áreas da Entomologia, como, por exemplo, em estudos sobre resistência de plantas, entomologia econômica e ecologia nutricional. Um dos fatores que pode interferir na quantidade de área foliar consumida pelo fitófago é a presença de certos químicos nas folhas, como a sinigrina em brassicáceas. Dessa forma, foram aplicadas em folhas de couve e brócolis diversas concentraçóes de sinigrina $(0,2,0,4$, $0,8,1,6$, e 3,2 mg $/ \mathrm{mL}$ em solução a $5 \%$ de Tween $20^{\circledR}$ ), medindo-se o consumo da área foliar por lagartas de P. xylostella, o qual foi aferido pela porcentagem de redução da área, diferença de peso e escala visual de notas. Os resultados mostraram que as concentrações 0,2 , 0,4 e $3,2 \mathrm{mg} / \mathrm{mL}$ de sinigrina aumentam o consumo em folhas de couve e diminuem em folhas de brócolis por lagartas de P. xylostella. As metodologias utilizadas proporcionam resultados similares, sendo as estimativas de peso fresco e nota visual mais práticas.

PALAVRAS-CHAVE: Plutella xylostella; glicosinolatos; Brassicaceae; crucíferas.

\begin{abstract}
Leaf area estimation, based on their reduction due to consumption by phytophagous insects, is a basic methodology in several areas of Entomology, for instance, in studies about plant resistance, economic entomology and nutritional ecology. One of the factors that can affect the amount of leaf area consumed by insects is the presence of certain chemicals in the leaves, such as sinigrin in Brassicaceae. Thus, several concentrations of sinigrin were applied on the leaves of cabbage and broccoli $(0.2,0.4$, $0.8,1.6$, and $3.2 \mathrm{mg} / \mathrm{mL}$ in $5 \%$ Tween $20^{\circledR}$ solution) in order to measure leaf area consumption by larvae of $P$. xylostella, estimated by the percentage of area reduction, weight loss and visual scale of notes. The results show that the concentrations of $0.2,0.4$ and 3.2 $\mathrm{mg} / \mathrm{mL}$ of sinigrin increase larvae consumption of $P$. xylostella on kale leaves and decrease it on broccoli leaves. The methodologies used provide similar results, and fresh weight measurements and visual grades are more practical.
\end{abstract}

KEYWORDS: Plutella xylostella; glucosinolates; Brassicaceae; cruciferous. 


\section{INTRODUÇÃO}

A traça-das-crucíferas, Plutella xylostella (L.) (Lep.: Plutellidae), é uma praga especialista encontrada em mais de 40 espécies de brássicas, causando danos e reduzindo significativamente a produção em diversas regiôes do mundo (MARAZZi et al., 2004; CHeng et al., 2008).

O método de controle mais utilizado para reduzir os danos desta praga é o químico. No entanto, os produtos químicos têm a capacidade de selecionar populações da traça-das-crucíferas resistentes aos produtos comerciais usualmente empregados (Castelo Branco; França, 2001). Por isso, técnicas alternativas de controle, como o uso de substâncias naturais das plantas, como o óleo de Neem (Azadirachta indica A. Juss.) que pode ter, por exemplo, ação repelente, antialimentar e tóxica para muitas espécies de insetos, têm sido avaliadas (TorRes et al., 2001; Mancebo et al., 2002; El Shafie; Basedow, 2003; BorçA JúNior et al., 2005). Essas substâncias têm como vantagens: seletividade, baixa toxicidade e eficiência contra várias espécies de insetos-praga (SCHMUtTerer, 1987; SaXena, 1989; Neves; Nogueira, 1996; Charleston et al., 2005).

Os glicosinolatos são um grupo de químicos secundários de plantas característicos na família Brassicaceae (Reichelt et al., 2002; MarAzZi et al., 2004; BARKER et al., 2006; CARTEA et al., 2011), que podem reduzir o ataque de insetos herbívoros (Van Poecke et al., 2003; Renwick et al., 2006). A sinigrina (2-propenylglucosinolate) pertence ao grupo dos glicosinolatos e ocorre naturalmente em plantas de brássicas, possuindo também propriedades anticarcinogênicas (SHELton; Nault, 2004; Halkier; Gershenzon, 2006; Martin; Müller, 2007; Velasco et al., 2007; Kehr; Buhtz, 2011; Peres, 2013).

É sabido que os glicosinolatos participam como estimulantes da alimentação e na seleção da planta hospedeira pelo inseto (SARFAZ et al., 2006; Hopkins et al., 2009). Entretanto, há ausência de informaçôes sobre o comportamento dessas substâncias no desenvolvimento dos insetos, o que requer estudos sobre a influência dos metabólitos secundários das plantas nos aspectos biológicos de pragas importantes, como a traça-das-crucíferas.

Gupta; Thorsteinson (1960) demonstraram que constituintes de plantas hospedeiras afetam a alimentação larval e a oviposição de P. xylostella. Mais tarde, estudos confirmaram que o reconhecimento do hospedeiro e a oviposição pelos insetos também dependem de glicosinolatos. Depois de encontrarem uma planta hospedeira, estímulos de contato podem influenciar a oviposição. No caso de P. xylostella, glicosinolatos individuais, inclusive sinigrina e glicobrassicina, desempenham papel importante no reconhecimento do hospedeiro e estímulo à oviposiçāo (ReED et al., 1989; RENwiCK, 2002). Afetam também a alimentação das larvas (VAN LoON et al., 2002).

Em brassicáceas, CaRvalHo et al. (2010) verificaram que a baixa concentração de sinigrina em couve e brócolis náo prejudica o desenvolvimento de $P$. xylostella, sendo que as altas concentraçôes afetam a viabilidade do inseto.

O consumo e a utilizaçâo de alimento constituem condiçóes primordiais para o crescimento, o desenvolvimento e a reprodução dos insetos, já que a quantidade e a qualidade do alimento utilizado na fase larval afetam o comportamento dos adultos (Souza et al., 2001). De acordo com Lara (1992), a quantidade e a qualidade dos alimentos interferem diretamente no ciclo biológico dos insetos, sendo que a perda da área foliar altera componentes do rendimento em decorrência das alteraçôes provocadas na fisiologia das plantas, refletindo na produtividade da planta (Moura, 1999).

O objetivo deste trabalho foi determinar o consumo foliar em couve (Brassica oleracea var. acephala DC, 1821) e brócolis (Brassica oleracea var. italica Plenck, 1794) por lagartas de $P$. xylostella quando diferentes concentraçôes de sinigrina foram aplicadas à superfície foliar dessas plantas.

\section{MATERIAL E MÉTODOS}

O trabalho foi desenvolvido no Laboratório de Biologia e Criação de Insetos (LBCI), da Faculdade de Ciências Agrárias e Veterinárias da Universidade Estadual Paulista (FCAV-UNESP), Jaboticabal (SP), em sala climatizada a $25 \pm 1{ }^{\circ} \mathrm{C}$, umidade relativa de $70 \pm 10 \%$ e fotoperíodo de 12 horas (12L:12E).

Utilizaram-se lagartas de segundo ínstar de P. xylostella, oriundas da criaçáo-estoque do laboratório, uma vez que lagartas de primeiro ínstar têm hábito minador e não teriam ingerido a sinigrina aplicada à superfície das folhas.

Foram utilizadas folhas de duas variedades de brássicas: couve-manteiga (B. oleracea var. acephala), variedade 'Da Geórgia' (Top Seed - Agristar), na qual não foi encontrada sinigrina em estudos anteriores (Thuler et al., 2007), e brócolis (B. oleracea var. italica), 'Ramoso Piracicaba Precoce' (Sementes Feltrin), que possui sinigrina naturalmente nas folhas $(0,02-0,04 \mu \mathrm{mol} / \mathrm{g})$ (RANGKADILOK et al., 2002a,b).

A sinigrina (Sigma) foi aplicada em discos de folhas de $2,2 \mathrm{~cm}$ de diâmetro confeccionados com o auxílio de um vazador metálico. Foram usadas as concentraçōes 0,$2 ; 0,4 ; 0,8 ; 1,6$; $3,2 \mathrm{mg} / \mathrm{mL}$ do composto em solução $5 \%$ de Tween $20^{\circledR}$ e, na testemunha, foi usada apenas a soluçáo $5 \%$ de Tween $20^{\circledR}$.

Para determinação da alíquota do peso seco, 50 discos das variedades foram pesados (frescos) e, em seguida, individualizados em sacos de papel de $200 \mathrm{~g}$, sendo levados à estufa a $65 \pm 5^{\circ} \mathrm{C}$ por 48 horas. Na sequência foram pesados novamente, para estimar o peso seco a ser correlacionado com o peso fresco, por meio de equaçóes obtidas pela regressão linear. Foram medidos e pesados 120 discos de folhas dos cultivares (2,2 cm de diâmetro), sendo que, para a determinação da área foliar e do peso fresco, utilizaram-se aparelho aferidor de área foliar LI3000 e balança analítica, respectivamente. 
Os discos foram então submetidos à aplicaçáo de sinigrina nas referidas concentraçóes.

Foram realizadas 20 repetiçóes, cada uma constando de uma placa de Petri ( $6 \mathrm{~cm}$ de diâmetro), contendo um disco de folha do cultivar onde foi aplicado sinigrina $(5,0 \mu \mathrm{L} /$ face do disco) com o auxílio de um pincel até o molhamento completo da superfície foliar. O disco foi colocado sobre papel filtro, levemente umedecido com água destilada. Sobre os discos foliares foram colocadas 10 lagartas de segundo ínstar de P. xylostella e, posteriormente, as placas foram vedadas com filme plástico para manter a umidade e evitar a fuga dos insetos. Foram mantidas assim por 26 horas, tempo suficiente para discriminação do consumo entre os tratamentos, o que foi determinado em teste preliminar.

Após esse período, as lagartas foram retiradas, e os discos, limpos de restos de excrementos, sendo pesados e submetidos à análise da área foliar. Como o equipamento de aferição da área foliar (LI3000) não contabilizava a área da película que ficava na folha na parte consumida, essas películas foram retiradas, fazendo-se nova medição da área foliar. Para determinação da área consumida, efetuou-se a subtração da área restante pela área total medida no início do teste.

Esses discos foram individualizados em sacos de papel $(200 \mathrm{~g})$, sendo então levados à estufa a $65 \pm 5^{\circ} \mathrm{C}$ para secagem durante 48 horas. Após secagem foram novamente pesados, para determinação do peso seco após a alimentação. Para determinação do peso seco consumido, realizou-se a subtração do peso seco restante pelo peso seco total, estimado pela equação de regressão linear da alíquota.

Os dados encontrados foram transformados em $(x+0,5)^{1 / 2}$, realizando-se a análise de variância pelo teste $\mathrm{F}$, sendo as médias comparadas pelo teste de Tukey a 5\% e submetidas à regressão polinomial, para determinar as curvas mais ajustadas e os respectivos coeficientes de determinação $\left(\mathrm{R}^{2}\right)$. Além disso, o consumo foliar também foi avaliado atribuindo-se notas, utilizando-se uma escala visual variando de 0 a 5 (Tabela 1). Os métodos utilizados para medir o consumo foliar foram correlacionados por meio da análise de regressão.

\section{RESULTADOS E DISCUSSÃO}

A alíquota do peso seco foi significativa na regressão linear para couve e para brócolis, indicando que, pela equação, pode-se estimar o peso seco total (y) através do peso fresco medido ( $\mathrm{x}$ ) (Figs. 1 e 2). $\mathrm{O} \mathrm{R}^{2}$ em couve foi de 0,869 e, em brócolis, de 0,529 , sendo este um pouco baixo devido ao grande número de pontos estimados; porém, o F foi altamente significativo, com 99\% de chance de acerto, indicando a importância e a veracidade da equação.

$\mathrm{O}$ peso fresco consumido em couve apresentou maiores índices nas concentraçóes extremas 0,2 (0,0498 g) e 3,2 mg/mL
Tabela 1. Escala visual estabelecida para atribuição de notas às desfolhas provocados nos discos foliares de couve e brócolis por lagartas de Plutella xylostella.

\begin{tabular}{lc} 
Desfolha & Nota \\
\hline Nenhuma & 0 \\
\hline 1 a $10 \%$ & 1 \\
\hline 11 a $25 \%$ & 2 \\
\hline 26 a $50 \%$ & 3 \\
\hline 51 a $75 \%$ & 4 \\
\hline 76 a $100 \%$ & 5 \\
\hline
\end{tabular}

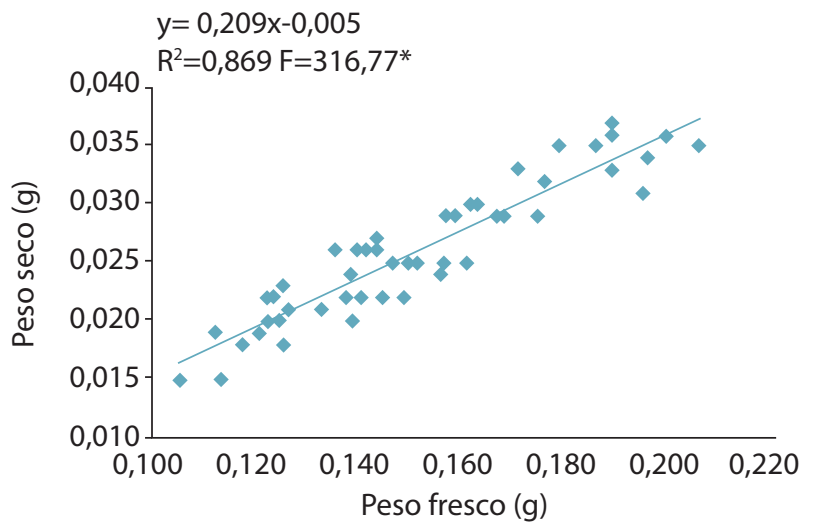

Figura 1. Curva de regressão obtida para as alíquotas de pesos fresco e seco de discos de discos de folhas de couve.

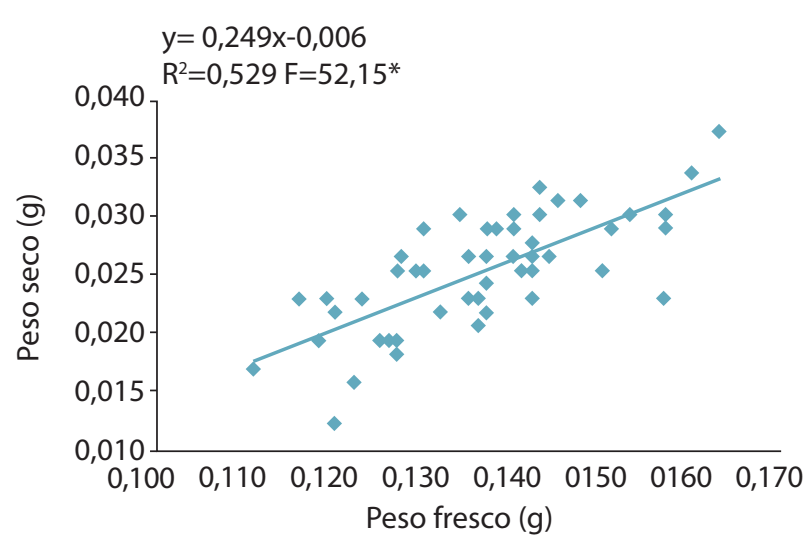

Figura 2. Curva de regressão obtida para as alíquotas de pesos fresco e seco de discos de discos de folhas de brócolis.

$(0,0516 \mathrm{~g})$ de sinigrina, diferindo significativamente das intermediárias (0,8 e 1,6 mg/mL), ajustando-se ao modelo quadrático estimado pela regressão (Tabela 2 e Fig. 3). Por outro lado, em brócolis, os maiores consumos foram verificados nas concentraçôes intermediárias de sinigrina $0,8(0,0419 \mathrm{~g})$ e $1,6 \mathrm{mg} / \mathrm{mL}$ $(0,0435 \mathrm{~g})$, que diferiram dos tratamentos com as menores $(0,2$ e $0,4 \mathrm{mg} / \mathrm{mL})$ (Tabela 2$)$. Isso resultou em uma parábola decrescente no modelo quadrático da regressão (Fig. 4), indicando, nesse caso, que as baixas concentraçóes de sinigrina 
Tabela 2. Peso fresco $(\mathrm{g})$, peso seco $(\mathrm{g})$ e área foliar $\left(\mathrm{cm}^{2}\right)$ consumidos e notas em função dos danos provocados por lagartas de Plutella xylostella nas diferentes doses de sinigrina $(\mathrm{mg} / \mathrm{mL})$.

\begin{tabular}{|c|c|c|c|c|c|c|c|c|}
\hline \multirow{3}{*}{$\begin{array}{l}\text { Tratamento* } \\
(\mathrm{mg} / \mathrm{mL})\end{array}$} & \multicolumn{4}{|c|}{ Peso Consumido ${ }^{1}$} & \multirow{2}{*}{\multicolumn{2}{|c|}{ Área Foliar Consumida' }} & \multirow{2}{*}{\multicolumn{2}{|c|}{ Notas }} \\
\hline & \multicolumn{2}{|c|}{ Fresco } & \multicolumn{2}{|c|}{ Seco } & & & & \\
\hline & Couve & Brócolis & Couve & Brócolis & Couve & Brócolis & Couve & Brócolis \\
\hline 0,0 & $0,0311 \mathrm{ab}$ & $0,0325 a b$ & $0,0061 b c$ & $0,0112 a b$ & $0,3680 b$ & $0,9205 a b$ & $2,65 b$ & $3,35 a b$ \\
\hline 0,2 & $0,0498 a$ & $0,0244 b$ & $0,0080 a b c$ & $0,0103 b$ & $0,4555 a b$ & $0,4480 c$ & $2,95 a b$ & $2,65 c$ \\
\hline 0,4 & $0,0364 a b$ & $0,0274 b$ & $0,0085 a b$ & $0,0103 b$ & $0,5620 a b$ & $0,4850 b c$ & $2,80 a b$ & $3, \mathrm{OObc}$ \\
\hline 0,8 & $0,0285 b$ & $0,0419 a$ & $0,0056 c$ & $0,0118 a b$ & $0,4120 b$ & $1,1060^{a}$ & $2,60 b$ & $3,60 a$ \\
\hline 1,6 & $0,0280 b$ & $0,0435 a$ & $0,0065 b c$ & $0,0135^{a}$ & $0,3000 b$ & $1,2725^{a}$ & $2,85 a b$ & $3,55 a b$ \\
\hline 3,2 & $0,0516 a$ & $0,0341 a b$ & $0,0102 a$ & $0,0127 a b$ & $0,7895 a$ & $0,5705 b c$ & $3,30^{a}$ & $3,35 a b$ \\
\hline $\mathrm{F}$ & $4,17^{* *}$ & $6,02 * *$ & $7,54 * *$ & $3,12^{*}$ & $4,71^{* *}$ & 8,89 ** & $3,00 *$ & $6,58^{* *}$ \\
\hline DMS & 0,0145 & 0,0087 & 0,0054 & 0,0021 & 0,1537 & 0,2027 & 0,5969 & 0,5790 \\
\hline CV (\%) & 2,15 & 1,30 & 2,47 & 0,32 & 17,19 & 19,52 & 22,76 & 19,42 \\
\hline
\end{tabular}

*Doses de sinigrina. ${ }^{1} A$ análise foi realizada com dados transformados em $(x+0,05)^{1 / 2}$; Médias seguidas da mesma letra na coluna não diferem entre si ao nível de $5 \%$.

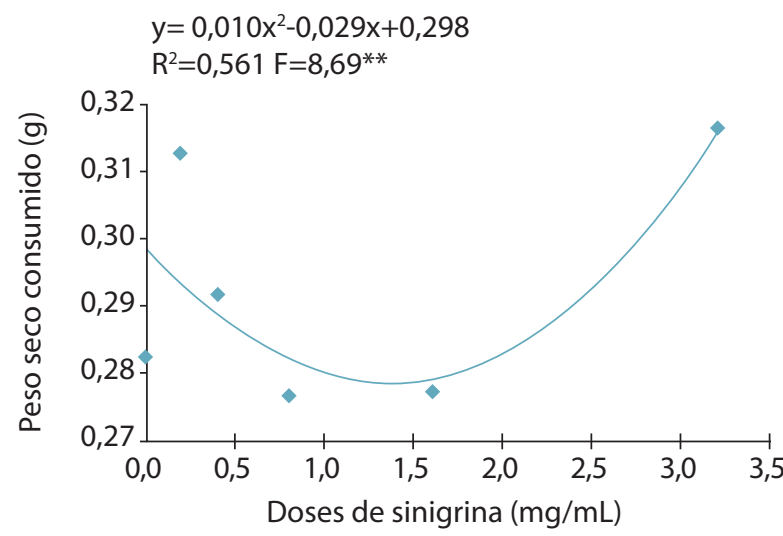

Figura 3. Curva ajustada para regressão polinomial entre o peso fresco consumido de folhas de couve por lagartas de Plutella xylostella e doses de sinigrina.

$(0,2$ e $0,4 \mathrm{mg} / \mathrm{mL})$ reduzem o consumo foliar. Esses dados contrariam os de THORSTEISON (1953), que afirmou ser a sinigrina atraente e estimulante de alimentação para $P$. xylostella, e também os de VAN LoON et al. (2002), que encontraram correlaçáo negativa na resposta comportamental de $P$. xylostella em repolho (B. oleraceae var. capitata), entre concentraçôes de sinigrina e intensidade de alimentação. Isso sugere que essas diferenças possam ser causadas por outros fatores, como a população e a idade das lagartas empregadas nos estudos, além das diferentes espécies/variedades/cultivares utilizadas. Também podem estar relacionadas às diferenças nos sistemas de produção das brassicáceas (por exemplo, adubação e sistemas de irrigação), condiçóes estas que podem alterar a composição química das plantas, afetando o desenvolvimento do inseto.

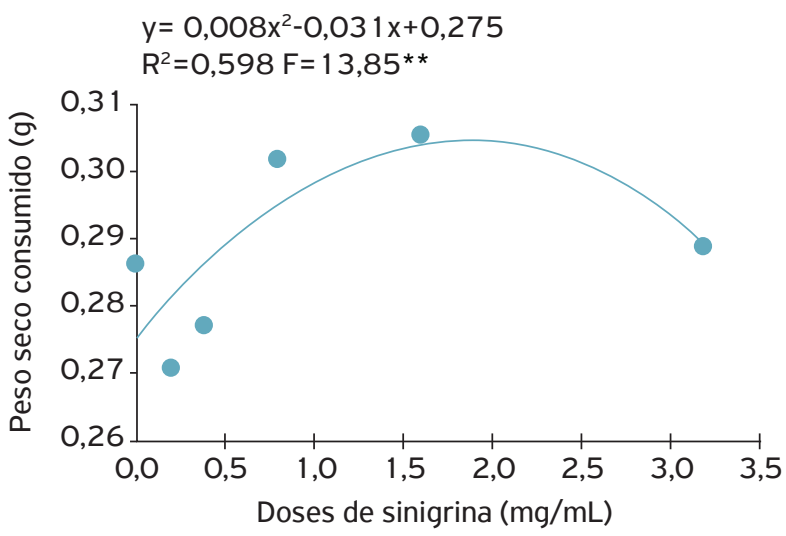

Figura 4. Curva ajustada para regressão polinomial entre o peso fresco consumido de folhas de brócolis por lagartas de Plutella xylostella e doses de sinigrina.

As medidas de peso seco em couve seguiram o mesmo padrão do peso fresco, sendo que o consumo foi maior nos extremos 0,2 (0,0080 g), 0,4 (0,0085 g) e 3,2 mg/mL (0,0102 g) de sinigrina (Tabela 2), ajustando-se à regressão polinomial de $2^{\circ}$ grau com parábola crescente (Fig. 5).

O mesmo não foi observado neste parâmetro para os tratamentos com folhas de brócolis, já que, como visto anteriormente no peso fresco, o maior consumo se deu no tratamento com concentração intermediária de sinigrina, $1,6 \mathrm{mg} / \mathrm{mL}$, apresentando $0,0135 \mathrm{~g}$ de peso seco médio consumido pelas largartas de P. xylostella, significativamente diferente das menores $(0,2$ e $0,4 \mathrm{mg} / \mathrm{mL}$ ) (Tabela 2 ). A curva mais ajustada para esta regressão foi a linear reta crescente. Neste caso, novamente as concentraçóes baixas de sinigrina, 0,2 e $0,4 \mathrm{mg} / \mathrm{mL}$, 


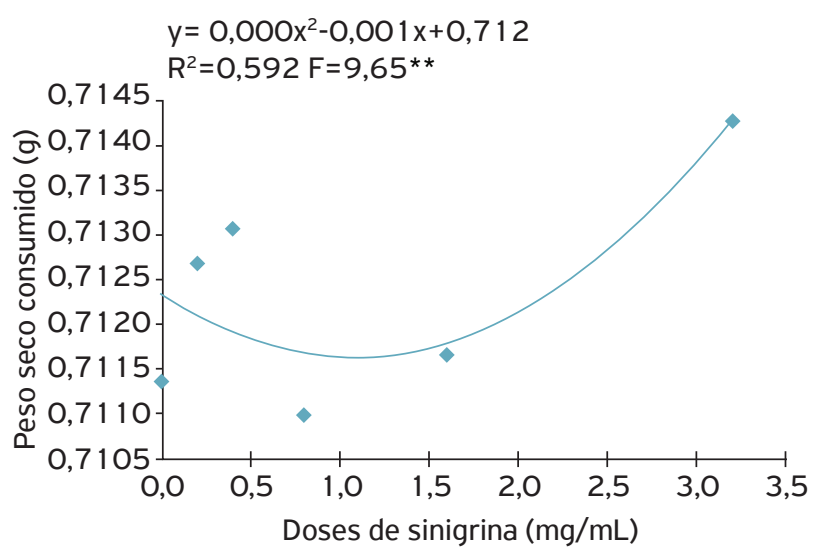

Figura 5. Curva ajustada para regressão polinomial entre o peso seco consumido de folhas de couve por lagartas de Plutella xylostella e doses de sinigrina.

prejudicaram o consumo, sendo menor que o tratamento controle, sem aplicação de sinigrina (Fig. 6).

Esses resultados são contrastantes com publicaçóes anteriores, comparando diferentes espécies de brássicas em níveis de glicosinolatos (Pivnick et al., 1994; BodnaryK, 1997). Esses autores concluíram que espécies como P. xylostella são insensíveis à sinigrina, e sugeriram que o status de praga em baixos níveis de glicosinolatos permaneceria inalterado, sendo que, para CARvalho et al. (2010), esses baixos níveis também não prejudicam o desenvolvimento do inseto; o mesmo não acontece com altos níveis, que alteram significativamente a viabilidade da praga.

A área foliar consumida em folhas de couve por lagartas de P. xylostella foi maior no tratamento com a concentraçáo mais elevada de sinigrina, $3,2 \mathrm{mg} / \mathrm{mL}$, sendo $0,7895 \mathrm{~cm}^{2}$ a área consumida, diferentemente dos tratamentos intermediários, de 0,8 e $1,6 \mathrm{mg} / \mathrm{mL}$, que apresentaram menores consumos $\left(0,4120\right.$ e $\left.0,300 \mathrm{~cm}^{2}\right)$, respectivamente (Tabela 2 e Fig. 7).

Nos tratamentos com folhas de brócolis, os resultados seguem o mesmo padrão dos demais parâmetros, sempre contrários aos apresentados com folhas de couve. Os maiores consumos ocorreram nas concentraçôes intermediárias de sinigrina, de 0,8 e 1,6 mg/mL, sendo $1,1060 \mathrm{~cm}^{2}$ e $1,2725 \mathrm{~cm}^{2}$, respectivamente, com consumo foliar nessas doses semelhantes à testemunha e diferentes das demais (Tabela 2 e Fig. 8).

Foi possível observar também que as lagartas alimentadas com folhas de brócolis e sinigrina apresentaram maior consumo foliar do que aquelas tratadas com folhas de couve e sinigrina, indicando uma possível preferência por aquela variedade (Tabela 2).

As notas estabelecidas em função dos danos provocados por lagartas de $P$. xylostella foram maiores nos tratamentos com folhas de brócolis do que em couve. Isso confirmou os parâmetros analisados, e brócolis foi o mais consumido pelas lagartas (Tabela 2).

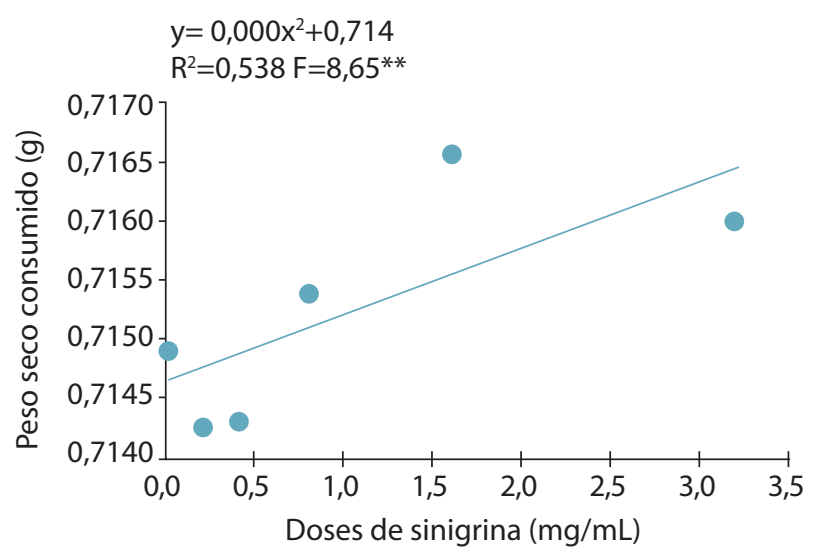

Figura 6. Curva ajustada para regressão polinomial entre o peso fresco consumido de folhas de brócolis por lagartas de Plutella xylostella e doses de sinigrina.

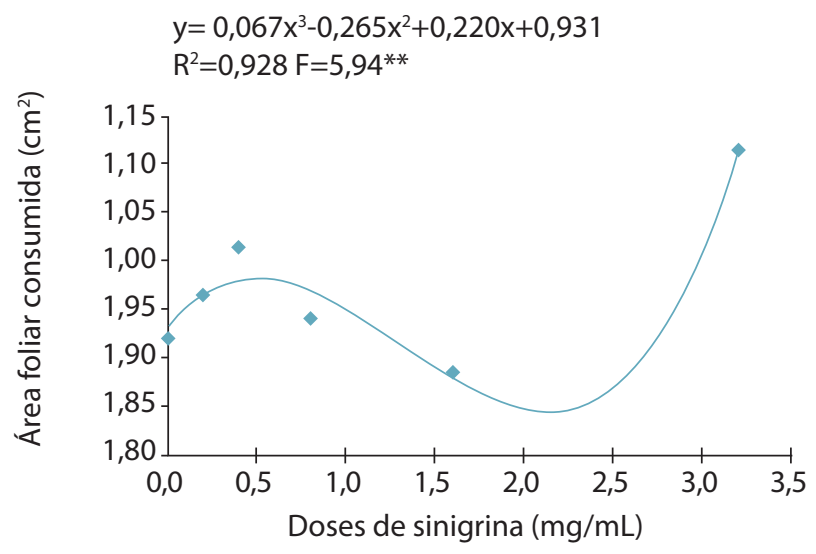

Figura 7. Curva ajustada para regressão polinomial entre a área foliar consumida de folhas de couve por lagartas de Plutella xylostella e doses de sinigrina.

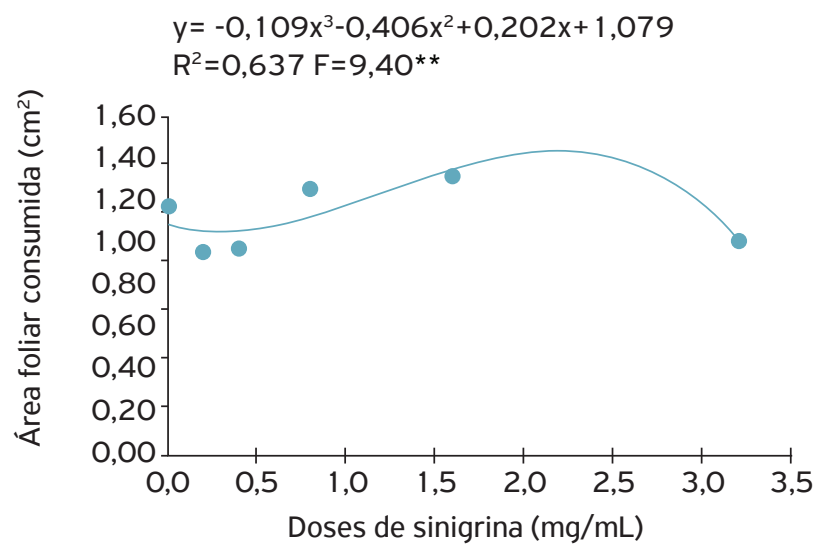

Figura 8. Curva ajustada para regressão polinomial entre a área foliar consumida de folhas de brócolis por lagartas de Plutella xylostella e doses de sinigrina.

Em couve, a curva que mais se ajustou aos dados na regressão foi a linear, enquanto que em brócolis foi a quadrática. Os valores das notas também seguiram o padrão de que, em couve, as 
concentraçóes de sinigrina extremas apresentaram maiores danos, enquanto em folhas de brócolis, os valores intermediários foram os mais danificados (Figs. 9 e 10). O R ${ }^{2}$ em brócolis, apesar de baixo $(0,346)$, foi significativo $\left(\mathrm{F}=6,28^{*}\right)$, respondendo a mais que seis vezes o valor do desvio da equação $(2,966)$. Além disso, do ponto de vista biológico, acredita-se que este gráfico seja uma reta, mas, devido a algum ponto fora da curva (outlier), a regressão quadrática também foi significativa (Fig. 10).

Nesse contexto, os resultados encontrados por ThorsteInson (1953), que testou a resposta larval de P. xylostella a três glicosinolatos (sinigrina, sinalbina e glicocheirolina), metabólitos secundários característicos da família Brassicaceae, mostram que esses produtos estimulam fortemente a alimentação de P. xylostella no quarto ínstar larval, tanto quando incorporados em dietas artificiais como em 18 plantas não hospedeiras, sendo as plantas completamente aceitas após a aplicação dos compostos.

A correlação dos métodos utilizados para medir o consumo foliar apresentou significância em couve para peso fresco e nota $\left(R=0,84^{*}\right)$, peso seco com área foliar $\left(R=0,87^{*}\right)$ e nota $\left(\mathrm{R}=0,86^{*}\right)$. Em brócolis, os métodos com correlaçâo significativa foram peso fresco com peso seco $\left(\mathrm{R}=0,87^{*}\right)$, área foliar $\left(0,93^{* *}\right)$ e nota $\left(\mathrm{R}=0,94^{* *}\right)$; área foliar e nota $\left(\mathrm{R}=0,84^{*}\right)$ (Tabela 3). Esses resultados mostram que as correlaçóes entre a maior parte dos métodos foram significativas, indicando alta similaridade entre os métodos utilizados para discriminar o consumo foliar. Dessa forma, a utilização de métodos mais práticos, como mediçôes de peso fresco e nota visual para o dano, torna-se mais eficiente.

As respostas encontradas para os pesos frescos e secos na área foliar consumida e, consequentemente, nas notas de danos, seguiram a mesma tendência. As concentraçóes extremas, de 0,2, 0,4 e 3,2 $\mathrm{mg} / \mathrm{mL}$ de sinigrina, apresentaram maior o consumo de folhas de couve e menor em brócolis, sendo que as lagartas nos tratamentos com brócolis preferiram concentraçôes intermediárias, como 0,8 e 1,6 mg/mL. Além disso, observou-se também, em praticamente todos os tratamentos, que o consumo foi maior pelas lagartas alimentadas com folhas de brócolis. Essa diferença nos dados obtidos para os tratamentos com folhas de couve e de brócolis deve estar ligada ao teor de sinigrina encontrado naturalmente nas folhas de brócolis e à sua ausência nas folhas de couve, que pode ter estimulado mais a alimentação pelas lagartas.

A variação no padrão de glicosinolatos tem sido atribuída a fatores genéticos e ambientais, incluindo idade da planta, temperatura, estresse hídrico e tipo de solo, sendo que a distribuição desses compostos varia com a parte da planta, com diferenças quantitativas e qualitativas no meio das raízes, folhas, talos e semente. Além disso, o ataque de pragas pode alterar notadamente o nível de glicosinolatos nas folhas (Velasco et al., 2007).

RANGKADILOK et al. (2002b) citaram a ocorrência de grande variação na concentração de sinigrina entre as espécies de brássicas, como B. oleraceae var. capitata, B. oleraceae var. botrytis, B. oleraceae var. itálica, B. oleraceae var. alboglabra, o hibrido B. oleraceae var. botrytis $\mathrm{x}$ B. oleraceae var. alboglabra, $B$. oleraceae var. gemminifera, $B$. nigra, $B$. juncea, B. rapa e $B$. napus, sendo que baixa concentração de sinigrina foi obtida no brócolis $(0,02-0,04 \mu \mathrm{mol} / \mathrm{g})$, quando comparada às altas concentraçôes encontradas no repolho e na couve-flor (23,79 e 23,69 $\mu \mathrm{mol} / \mathrm{g}$, respectivamente), citadas por aqueles autores. A ausência desta substância em couve comparada à sua presença em brócolis pode ter sido fundamental para as diferenças nos resultados obtidos nas variáveis testadas. Por outro lado, THuLER et al. (2007) não encontraram sinigrina em diversas variedades de brássicas, incluindo a couve-manteiga 'Da Geórgia' utilizada neste trabalho.

VeLASCO et al. (2007) concluíram que o padráo de glicosinolato foi estável nas partes da planta, mas não nas diferentes fases do desenvolvimento. Brotos floridos e folhas colhidas cinco meses depois do transplante continham concentração mais alta de glicosinolatos, em particular sinigrina. Os glicosinolatos alifáticos (composto orgânico que năo é cíclico) foram menos suscetíveis aos efeitos ambientais do que os glicosinolatos indol

$$
\begin{aligned}
& y=-0,164 x+2,688 \\
& R^{2}=0,615 F=9,23 * *
\end{aligned}
$$

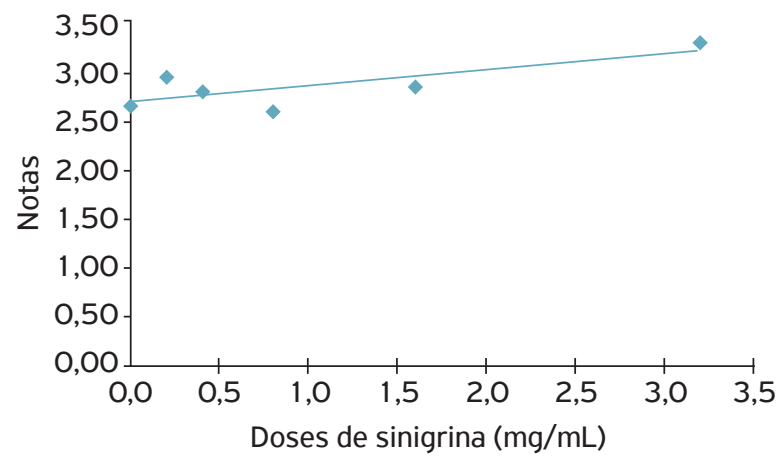

Figura 9. Curva ajustada para regressão polinomial entre as notas em função dos danos nas folhas de couve provocados por lagartas de Plutella xylostella e doses de sinigrina.

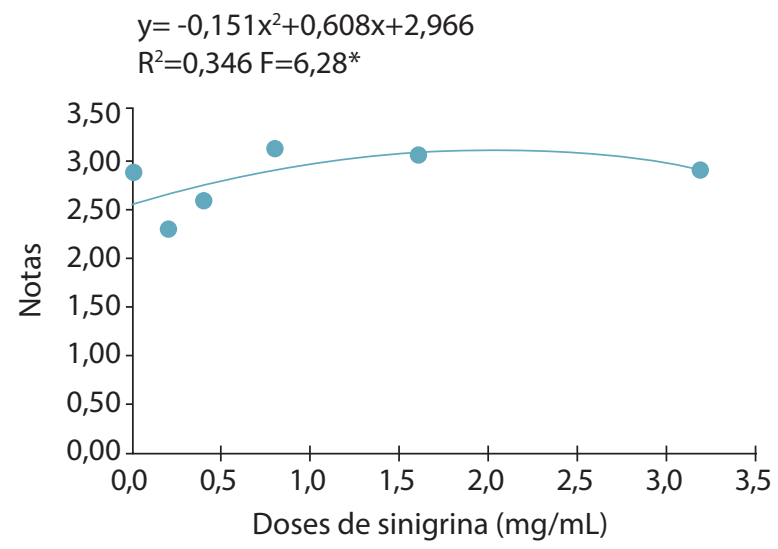

Figura 10. Curva ajustada para regressão polinomial entre as notas em função dos danos nas folhas de brócolis provocados por lagartas de Plutella xylostella e doses de sinigrina. 
Tabela 3. Coeficiente de correlação $(R)$ e teste $T$ para $R(p \leq 0,05)$ entre os métodos utilizados para avaliar o consumo foliar e o dano provocado pelas lagartas de Plutella xylostella em folhas de couve e brócolis com sinigrina.

\begin{tabular}{lcccccc} 
& \multicolumn{3}{c}{ Couve } & \multicolumn{3}{c}{ Brócolis } \\
\cline { 2 - 7 } & Peso seco & Área foliar & Nota & Peso seco & Área foliar & Nota \\
\hline Peso fresco & $0,77^{\text {n.s. }}$ & $0,76^{\text {n.s. }}$ & $0,84^{*}$ & $0,87^{*}$ & $0,93^{* *}$ & $0,94^{* *}$ \\
\hline Peso seco & - & $0,87^{*}$ & $0,86^{*}$ & - & $0,68^{\text {n.s. }}$ & $0,78^{\text {n.s. }}$ \\
\hline Área foliar & - & - & $0,78^{\text {n.s. }}$ & - & - & $0,84^{*}$ \\
\hline
\end{tabular}

(composto orgânico heterocíclico). A relação entre a variação na concentração de glicosinolatos em folhas e o dano causado por várias espécies de lepidópteros especialistas parece ocorrer, embora seja difícil confirmar uma relação direta entre a perda de conteúdo de glicosinolatos em folhas e o dano da praga. Os fatores ambientais, como $\mathrm{pH}$ do solo e temperatura, parecem ter alguma influência na variação de glicosinolatos.

\section{CONCLUSÕES}

As concentraçôes $0,2,0,4$ e 3,2 $\mathrm{mg} / \mathrm{mL}$ de sinigrina aumentaram o consumo em folhas de couve e diminuíram o consumo de folhas de brócolis por lagartas de P. xylostella. As mediçóes de peso fresco e nota visual são as mais práticas e menos custosas para avaliar o consumo das lagartas de P. xylostella.

| | | | | | | | | | | | | | | | | | | | | | | | | | | | | | | | | | | | | | | | | | | | | | | | | | | | | | | | | | | | | | | | | | | | | | | | | | | | | | | | | | | | | | | | | | | | | | | | | | | | | | | | | | | | | | | | | | | | | | | | | | | | | | | | | | | | | | | | | | | | | | | | | | | | | | | | | | | | | | | | | | | | | | | | | | | | | | | | | | | | | | | | | | | | | | | | | | | | | | | | | | | | | | | | | | | | | | | | | | | | | |

\section{REFERÊNCIAS}

BARKER, A.M.; MOLOTSANE, R.; MÜLLER, C.; SCHAFFNER, U.; STÄDLER, E. Chemosensory and behavioral responses of the turnip sawfly, Athalia rosae, to glucosinolates and isothiocyanates. Chemoecology, v.16, n.4, p.209-218, 2006.

BODNARYK, R.P. Will low-glucosinalate cultivar of the mustards Brassica juncea and Sinapis alba be vulnerable to insect pests? Canadian Journal of Plant Science, v.77, n.2, p.283-287, 1997

BOIÇA JÚNIOR, A.L.; MEDEIROS, C.M.A.; TORRE, A.L.; CHAGAS FILHO, N.R. Efeito de extratos aquosos de plantas no desenvolvimento de Plutella xylostella (L.) (Lepidoptera: Plutellidae) em couve. Arquivos do Instituto Biológico, v.72, p.45-50, 2005.

CARVALHO, J.S.; DE BORTOLI, S.A.; THULER, R.T.; GOULART, R.M.; VOLPE, H.X.L. Efeito de sinigrina aplicada em folhas de brássicas sobre características biológicas de Plutella xylostella (L.) (Lepidoptera: Plutellidae). Acta Scientiarum. Agronony, v.32, n. 1 , p. 15-20, 2010.

CASTELO BRANCO, M.; FRANÇA, F.H. Traça-das-crucíferas, Plutella xylostella (Lepidoptera: Yponomeutidae). In: VILELA, E.F.; ZUCCHI, R.A.; CANTOR, F. (Eds.). Histórico e impacto das pragas introduzidas no Brasil. Ribeirão Preto: Holos, 2001. p.85-89.

CHARLESTON, D.S.; KFIR, R.; VET, L.E.M.; DICKE, M. Behavioural responses of diamondback moth Plutella xylostella (Lepidoptera: Plutellidae) from Melia azedarach and Azadirachta indica. Bulletin of Entomological Research, v.95, n.5, p.457-465, 2005.

CHENG, L.; YU, G.; CHEN, Z.; LI, Z. Insensitive acetylcholine receptor conferring resistance of Plutella xylostella to nereistoxin insecticides. Agricultural Sciences in China, v.7, n.7, p.847-852, 2008.

EL SHAFIE, H.A.F.; BASEDOW, T. The efficacy of different neem preparations for the control of insects damaging potatoes and plants in the Sudan. Crop Protection, v.22, n.8, p.1015-1021, 2003.
GUPTA, P.D.; THORSTEINSON, A.J. Food plant relationships of the diamondback moth (Plutella maculipennis (Curt)). Entomologia Experimentalis et Applicata, v.3, n.3, p.241-250, 1960.

HALKIER, B.A.; GERSHENZON, J. Biology and biochemistry of glucosinolates. Annual Review of Plant Biology, v.57, p.303333, 2006.

HOPKINS, R.J.; VAN DAM, N.M.; VAN LOON, J.J.A. Role of glicosinolates in insect-plant relationships and multitrophic interactions. Annual Review of Entomology, v.54, n. 1, p.5783, 2009.

KEHR, J.; BUHTZ, A. Brassica proteomics and metabolomics. In: EDWARDS, D.; BATLEY, J.; KOLE, C. (Eds.). Genetics, genomics and breeding of oilseed brassicas. Enfield: Science Publishers, 2011. p.174-193.

LARA, F.M. Princípios de entomologia. 3a ed. São Paulo: Ícone, 1992. $331 \mathrm{p}$.

MANCEBO, F.; HILJE, L.; MORA, G.A.; SALAZAR, R. Biological activity of two nem (Azadirachta indica A. Juss., Meliaceae) products on Hypsipyla grandella (Lepidoptera: Pyralidae) larvae. Crop Protection, v.21, p.107-112, 2002.

MARAZZI, C.; PATRIAN, B.; STÄDLER, E. Secondary metabolites of the leaf surface affected by sulphur fertilization and perceived by the diamondback moth. Chemoecology, v.14, n.2, p.81-86, 2004.

MARTIN, N.; MÜLLER, C. Induction of plant responses by a sequestering insect: Relationship of glucosinolate concentration and myrosinase activity. Basic and Applied Ecology, v.8, p.1325, 2007.

MOURA, G.M. Efeitos do desfolhamento no rendimento do feijoeiro. Pesquisa Agropecuária Brasileira, v.34, n. 1, p.57-62, 1999. 
NEVES, B.P.; NOGUEIRA, J. C. M. Cultivo e utilização do nim indiano (Azadirachta indica A. Juss.). Embrapa-CNPAF - APA, 1996. 32p. (Circular Técnica, 28).

PERES, L.E.P. Metabolismo secundário. Pelotas: UFPEL. Disponível em: <http://www.ufpel.edu.br/biotecnologia/gbiotec/site/ content>. Acesso em: 8 ago. 2013.

PIVNICK, K.A.; JARVIS, B.J.; SLATER, G.P. Identification of olfactory cues used in host-plant finding by diamondback moth, Plutella xylostella (Lepidoptera: Plutellidae). Journal of Chemical Ecology, v.20, n.7, p.1407-1427, 1994.

RANGKADILOK, N.; NICOLAS, M.E.; BENNETT, R.N.; PREMIER, R.R.; EAGLING, D. R.; TAYLOR, P.W.J. Developmental changes of sinigrina and glucoraphanin in three Brassica species (Brassica nigra, Brassica juncea and Brassica oleracea var. italica). Scientia Horticulturae, v.96, n.1, p.11-26, 2002a.

RANGKADILOK, N.; NICOLAS, M.E.; BENNETT, R.N.; PREMIER, R.R.; EAGLING, D.R.; TAYLOR, P.W.J. Determination of sinigrina and glucoraphanin in Brassica species using a simple extraction method combined with ion-pair HPLC analysis. Scientia Horticulturae, v.96, n. 1, p. $27-41,2002 b$.

REED, D.W.; PIVNICK, K.A.; UNDERHILL, E.W. Identification of chemical oviposition stimulants for the diamondback moth, Plutella xylostella, present in three species of Brassicaceae. Entomologia Experimentalis et Applicata, v.53, n.3, p.227-286, 1989.

REICHELT, M.; BROWN, P.D.; SCHNEIDER, B.; OLDHAM, N.J.; STAUBER, E.; TOKUHISA, J.; KLIEBENSTEIN, D.J.; MITCHELLOLDS, T.; GERSHENZON, J. Benzoic acid glucosinolate esters and other glucosinolates from Arabidopsis thaliana. Phytochemistry, v.59, n.6, p.663-671, 2002.

RENWICK, J.A.A. The chemical world of crucivores: lures, treats and traps. Entomologia Experimentalis et Applicata, v.104, n.1, p.35-42, 2002.

SARFAZ, M.; DOSDALL, L.M.; KEDDIE, B.A. Diamondback moth-host plant interactions: implications for pest management. Crop Protection, v.25, n.7, p.625-639, 2006.
SAXENA, R.C. Inseticides from Neem. In: ARNASON, J.T.; PHILOGENE, B.J.R.; MORAND, P. (Eds.). Inseticides of plant origin. Washington: American Chemical Society, p.110-129, 1989.

SCHMUTTERER, $\mathrm{H}$. Insect growth-disrupting and fecundity-reducing ingredients from the neem and chynaberry trees. In: MORGAN, E.D.; MANDAVA, N.B. (Eds.). CRC Handbook of natural pesticides: volume III, Insect Growth Regulators - PartB. Washington: CRC, p.119-167, 1987.

SHELTON, A.M.; NAULT, B.A. Dead-end trap cropping: a technique to improve management of the diamondback moth, Plutella xylostella (Lepidoptera: Plutellidae). Crop Protection, v.23, n.6, p.497-503, 2004.

SOUZA, A.M.L.; ÁVILA, C.J.; PARRA, J.R.P. Consumo e utilização de alimento por Diatraea saccharalis (Fabr.) (Lepidoptera: Pyralidae), Heliothis virescens (Fabr.) e Spodoptera frugiperda (J. E. Smith) (Lepidoptera: Noctuidae) em duas temperaturas. Neotropical Entomology, v.30, n.1, p.11-17, 2001.

THORSTEINSON, A.J. The chemotactic responses that determine host specificity in an oligophagous insect (Plutella maculipennis) (Curt.) (Lepidoptera). Canadian Journal of Zoology, v.31, n. 1, p.52-72, 1953.

THULER, R.T.; DE BORTOLI, S.A.; HOFFMANN-CAMPO, C.B. Classificação de cultivares de brássicas com relação à resistência à traça-das-crucíferas e à presença de glucosinolatos. Pesquisa Agropecuária Brasileira, v.42, n.4, p.467-474, 2007.

TORRES, A.L.; BARROS, R.; OLIVEIRA, J.R. Efeito de extratos aquosos de plantas no desenvolvimento de Plutella xylostella (L., 1758) (Lepidoptera: Plutellidae). Neotropical Entomology, v.30, n. 1, p.151-156, 2001.

VAN LOON, J.J.A.; WANG, C.Z.; NIELSEN, J.K.; GOLS, R.; QIU, Y.T. Flavonoids from cabbage are feeding stimulants for diamondback moth larvae additional to glucosinolatos: chemoreception and behavior. Entomologia Experimentalis et Applicata, v. 104, n. 1, p.27-34, 2002.

VELASCO, P.; CARTEA, M.E.; GONZÁLEZ, C.; VILAR, M.; ORDÁS, A. Factors affecting the glucosinolate content of kale (Brassica oleraceae acephala group). Journal of Agricultural and Food Chemistry, v.55, n.3, p.955-962, 2007. 